\title{
Editorial: Re-Enacting Sensorimotor Experience for Cognition
}

\author{
Guido Schillaci ${ }^{*}$, Verena V. Hafner ${ }^{1}$ and Bruno Lara ${ }^{2}$ \\ ${ }^{1}$ Adaptive Systems Group, Department of Computer Science, Humboldt-Universität zu Berlin, Berlin, Germany, ${ }^{2}$ Cognitive \\ Robotics Group, Center for Science Research, Universidad Autonoma del Estado de Morelos, Cuernavaca, Mexico
}

Keywords: sensorimotor simulations, cognitive development in robots, prospection, sensorimotor experience, internal models

\section{Editorial on the Research Topic}

Re-Enacting Sensorimotor Experience for Cognition

\section{OPEN ACCESS}

Edited and Reviewed by: Jochen J. Steil,

Braunschweig University of

Technology, Germany

*Correspondence: Guido Schillaci guido.schillaci@informatik.hu-berlin.de

Specialty section: This article was submitted to Humanoid Robotics,

a section of the journal

Frontiers in Robotics and Al

Received: 15 October 2016 Accepted: 12 December 2016 Published: 23 December 2016

Citation:

Schillaci G, Hafner VV and Lara B (2016) Editorial: Re-Enacting Sensorimotor Experience for Cognition.

Front. Robot. Al 3:77. doi: 10.3389/frobt.2016.00077
Recent findings in cognitive science suggest that the human brain implements processes of simulation of sensorimotor activity (Pezzulo et al., 2013; Case et al., 2015; Wood et al., 2016). By re-enacting sensorimotor experience, the brain would be capable of anticipating the sensory consequences of intended motor actions. This would enable the individual to efficiently and fluidly interact with the environment.

This e-book puts forward the hypothesis that similar mechanisms underlie the development of basic cognitive capabilities. Therefore, sensorimotor simulation processes may represent one of the bridges between motor development and cognitive development in humans.

This collection comprises manuscripts published by Frontiers in Robotics and Artificial Intelligence, under the section Humanoid Robotics in the research topic "Re-enacting sensorimotor experience for cognition." The e-book aims at condensing the latest theoretical review and experimental studies that address new paradigms for learning and integrating multimodal sensorimotor information in artificial agents, re-use of the sensorimotor experience for cognitive development and further construction of more complex strategies and behaviors using these concepts.

\section{THEORETICAL AND REVIEW STUDIES}

In their review paper, Schillaci et al. introduce recent research on exploration as a drive for motor and cognitive development, and how this has been applied to robotics. After focusing on the development of internal body representations, the authors review research that highlights the importance of sensorimotor simulations and their role in the grounding of higher cognitive capabilities in robots. Most of these works have been inspired by sensorimotor and enactive theories. Froese and Sierra, in their review of the volume edited by Bishop and Martin on Contemporary Sensorimotor Theory (2014), draw the attention of the reader to the similarities and the differences of the current sensorimotor and enactive theories. However, the authors point out the need of additional comparative studies, in particular in the context of Robotics and AI.

Nonetheless, several challenges have already been posed by these theories. How can we explain the phenomenological character of experience (Froese and Sierra)? Are body representation and internal simulation processes involved in coding a basic sense of self in artificial agents, and if 
so, how (Schillaci et al.; Schillaci et al., 2016)? What should be built into an artificial agent "so that it really feels the touch of a finger, the redness of red, or the hurt of a pain" (O'Regan, 2014)? Terekhov and O'Regan show mathematically and in simulation that naive artificial agents can build the abstract notion of space from their perceptual systems by learning sensorimotor invariants. Without making assumptions about the existence of space, such agents are able to learn the notion of rigid displacement. Their findings give a role to artificial intelligence in the quest of explaining the nature of space, prevalently addressed by philosophy and physics.

Vernon et al. analyze the role of memory in anticipation processes. They propose a framework integrating procedural and episodic memory into internal simulation processes. Joint episodic and procedural memory facilitates prospection as it constrains the combinatorial explosion of potential perceptionaction associations allowing effective action selection in reaching goals.

\section{EXPERIMENTAL STUDIES}

Motor and cognitive developments in infancy are characterized by a process, where the individual is actively involved in the shaping of the experience through exploration (Schillaci et al.). Exposure to different sensorimotor experiences can influence cognitive development. Lones et al. demonstrate that this applies also to artificial agents. They observed that robots showed greater cognitive capabilities when exposed to a rich set of novel sensorimotor experiences as opposed to robots raised in poorly stimulant environments.

Exploration is the drive for motor and cognitive development (Schillaci et al.). The production of behavioral diversity is fundamental for discovering new aspects of the environment and of the individuals embodiment. Benureau and Oudeyer propose a mechanism for creating behavioral diversity in robots by re-enacting previously experienced sensorimotor actions. The artificial agent is capable of learning efficiently and adapting its past experience to new contexts even when characterized by high dimensional sensorimotor spaces. For example, by re-enacting past interaction with an object, the system is capable of learning more efficiently and capable of adapting to objects that are different in morphology.

How can this sensorimotor experience be stored in artificial agents? Vicente et al. propose a mechanism for simultaneous body schema adaptation and end-effector pose estimation on a humanoid robot. The system learns an internal body representation which is used to generate hypotheses of limb positions in space. These hypotheses are combined in a Bayesian fashion with the real perceptual feedback in a visual servoing control scheme that enables precise reaching actions.

Similarly, Escobar Juárez et al. address the development of internal body representations in artificial agents through
Self-Organizing Maps and Hebbian learning. The authors present two experiments that show the capabilities of this architecture to implement internal simulation processes. Most importantly, these experiments show its potential as a building block for the coding of more complex sensorimotor schemes and behaviors. Schrodt and Butz propose a similar architecture, but with a probabilistic flavor. The system they propose is based on a stochastic generative neural network and is capable of implementing mental simulations in an artificial system. Moreover, the system learns to infer actions from partial sensory information and implements imagination capabilities to emulate and to recognize observed actions using the self body model.

Finally, Billing et al. present a learning mechanism that enables a simulated robot to mentally imagine navigating in an apartment environment. The proposed model can learn from human demonstrations and can re-enact the demonstrated behavior both overtly (using real sensory observations) and covertly (using simulated sensory observations).

\section{CONCLUSION}

The studies presented in this e-book offer support for the fundamental role that processes of re-enacting sensorimotor experience can have in the development of motor and cognitive skills, both in humans and in artificial agents. By bringing together theoretical review and experimental studies, we hope to further strengthen what we believe to be a very important research topic.

The works presented here study highly important but still very low-level processes. A fundamental issue to be addressed in future research is how the behavioral and computational components identified by the aforementioned studies support higher level cognition and action. In fact, the majority of the studies in the field address the bootstrapping of particular skills without explaining how the development of further skills may progress. We strongly believe that further investigation on the interaction between mechanisms for artificial curiosity, exploration, body representations, memory, and simulation processes would provide insights in the quest for open-ended development in artificial agents.

\section{AUTHOR CONTRIBUTIONS}

Each of the authors has contributed equally and significantly to the study.

\section{FUNDING}

The work of GS and $\mathrm{VH}$ has been conducted as part of the EARS (Embodied Audition for RobotS) Project, which received funding from the European Union's Seventh Framework Programme (FP7/2007-2013) under grant agreement number 609465. 


\section{REFERENCES}

Case, L. K., Pineda, J., and Ramachandran, V. S. (2015). Common coding and dynamic interactions between observed, imagined, and experienced motor and somatosensory activity. Neuropsychologia 79, 233-245. doi:10.1016/j. neuropsychologia.2015.04.005

O'Regan, J. K. (2014). “The explanatory status of the sensorimotor approach to phenomenal consciousness, and its appeal to cognition," in Contemporary Sensorimotor Theory, eds J. M. Bishop, and A. O. Martin (Springer), 23-35.

Pezzulo, G., Candidi, M., Dindo, H., and Barca, L. (2013). Action simulation in the human brain: twelve questions. New Ideas Psychol. 31, 270-290. doi:10.1016/j. newideapsych.2013.01.004

Schillaci, G., Ritter, C. N., Hafner, V. V., and Lara, B. (2016). "Body representations for robot ego-noise modelling and prediction, towards the development of a sense of agency in artificial agents," in International Conference on the Simulation and Synthesis of Living Systems (ALife XV), (Cancún), 390-397.

Wood, A., Rychlowska, M., Korb, S., and Niedenthal, P. (2016). Fashioning the face: sensorimotor simulation contributes to facial expression recognition. Trends Cogn. Sci. 20, 227-240. doi:10.1016/j.tics.2015.12.010

Conflict of Interest Statement: The authors declare that the research was conducted in the absence of any commercial or financial relationships that could be construed as a potential conflict of interest.

Copyright (C) 2016 Schillaci, Hafner and Lara. This is an open-access article distributed under the terms of the Creative Commons Attribution License (CC BY). The use, distribution or reproduction in other forums is permitted, provided the original author(s) or licensor are credited and that the original publication in this journal is cited, in accordance with accepted academic practice. No use, distribution or reproduction is permitted which does not comply with these terms. 